\title{
VARIA \\ TRANSCRIPCIÓN DEL MANUSCRITO AUTÓGRAFO DE JUAN DE HERRERA SOBRE LA TEORIA MATEMÁTICA DE LAS GRUAS \\ (En el IV Centenario de la muerte de Juan de Herrera)
}

\section{El manuscrito de la Colección Pedraja}

Tiene interés el manuscrito que comentamos, conservado en la Biblioteca Municipal de Santander ${ }^{1}$ y con letra de principios del siglo actual, porque su título expresa el auténtico contenido del texto que escribió personalmente el arquitecto escurialense Juan de Herrera ${ }^{2}$, probablemente mediado el año 1575 en El Escorial ${ }^{3}$.

La portada de este manuscrito es la siguiente:

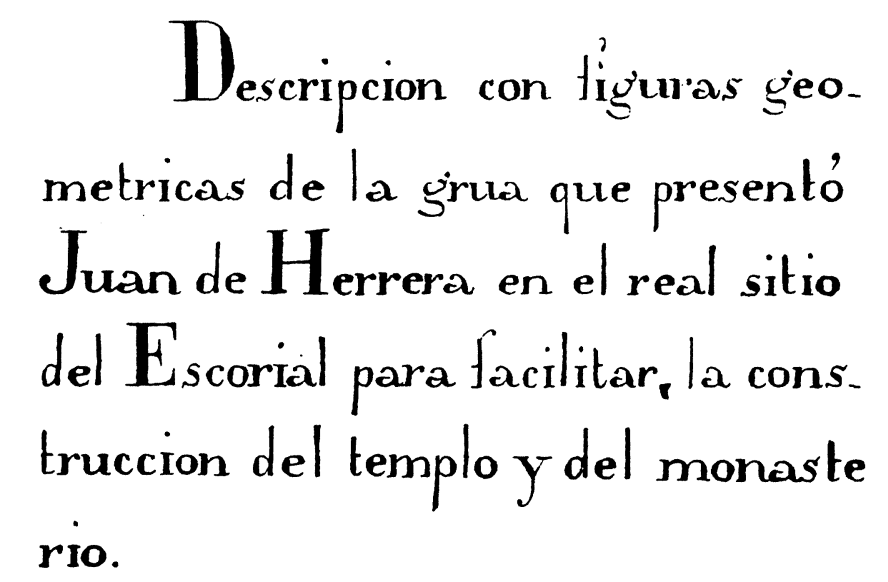

Fig. 1. Facsímil de la portada, reproducida al setenta por ciento de su tamaño.

\footnotetext{
1 Biblioteca Municipal en Santander, Colección E. de la Pedraja: Ms. 69 (17.2.12).

2 Cervera Vera: El manuscrito de Juan de Herrera, 12-14, sobre los análisis de la letra de Juan de Herrera.

3 Ibidem 15 y 16-17, se aportan datos cronológicos acerca de ambas circunstancias.
} 
Su texto transcribe con apreciable fidelidad el original de Herrera. Se caracteriza por su presentación correcta y caligrafía limpia con letra corriente. Los márgenes están justificados a derecha e izquierda de cada una de sus hojas, logrando uniformidad entre lineas y dejando los espacios necesarios en los cuales copia las figuras geométricas dibujadas por nuestro arquitecto para demostrar su teoría.

El ejemplar consta de portada y de siete hojas numeradas modernamente a lápiz. La medida de cada hoja es de $205 \times 145$ mm, mientras que la mancha de escritura tiene 14 ó 15 líneas y aproximadamente mide $190 \times 90 \mathrm{~mm}$, aunque el resto de la primera es menor, por comenzar su texto debajo de un espacio en blanco con una cruz sobre la dedicatoria a

\section{$C$ [atólica] $R$ [eal] $M$ [agestad] de Felipe II.}

En el resto del último folio solo se encuentra el esquema geométrico final y la anotación: $A r$ chivo gral de Simancas. / Obras y Bosques - Escorial. Leg. 2

$$
\begin{aligned}
& \text { - braco de toda la fromana y el euerjor } \\
& \text { c. es el pilon. ía potencia quasta in } \hat{\ell} \\
& \text { cabo de la aíngy rime } j \\
& \text { it averso 6. Lo que pe levanta (c) } \\
& \text { ¿y al pento a. la enpatado. } \\
& \text { de caiga. La Licha alcaprima ol' feil donde. } \\
& \text { cuelga el braso de ia irmana todo to yneat } \\
& \text { conta en la - jigura dida. }
\end{aligned}
$$

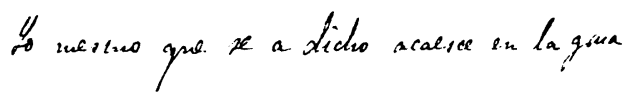

$$
\begin{aligned}
& \text { porque zuendo sn piriajal soimbro vne sue- }
\end{aligned}
$$

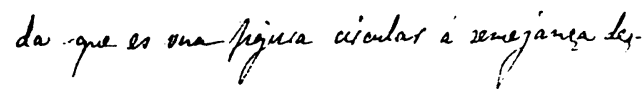

$$
\begin{aligned}
& \text { ta. pue aque se bee seinalada con estar letias } \\
& \text { a.c.d.e.f. la qual fijais } \\
& \text { un are de giesta groservico } \\
& \text { mo el que esta deneripto". }
\end{aligned}
$$

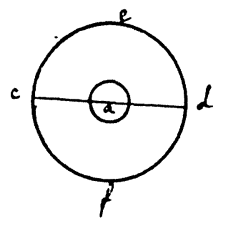

Fig. 2. Facsímil del texto de la página 4, reproducido al setenta por ciento de su tamaño. 
El copista del manuscrito de Juan de Herrera leyó cuidadosamente el texto original de Simancas ${ }^{4}$ y se refirió exclusivamente a la teoría de la grúa, como figura en la portada que reproducimos. Tiene por tanto valor esta transcripción pues únicamente aprecia el fundamento físico de igual ingenio.

\section{El original de Juan de Herrera}

El manuscrito del arquitecto escurialense está dirigido a Felipe II ${ }^{5}$, y es valioso por su contenido científico e importante por su valor histórico y humano.

Su contenido científico muestra la aplicación práctica de los conocimientos matemáticos de Juan de Herrera, redactados mediante sucesivos y sencillos conceptos que le conducen a explicar el de la grúa, para su fácil entendimiento por Felipe II.

Su valor histórico y humano es importante en dos aspectos. Uno, por haberlo escrito Juan de Herrera personalmente y con letra cuidadosa para el rey. Otro, porque el poderoso monarca, siempre ocupado en complejos problemas de Estado, lo mantuvo en sus regias manos cuando lo recibió y mientras lo estaba leyendo, para después, satisfecho, aprobarlo (Fig. 3).

Juan de Herrera expone sus teorías en este autógrafo de tres hojas de texto, escritas en su recto y verso, con una presentación magnífica y con caligrafía muy cuidada.

Como portada figura otra hoja en la que encontramos la abreviatura del apellido de nuestro arquitecto (Fig. 4).

$$
\text { herr. }^{a}
$$

y el título:

\section{Architetura y machinas.}

En esta misma portada se encuentran las dos iniciales O. y B., a las que sigue la numeración antigua (núm. 63), actualmente tachada. A continuación el sello estampado del Archivo General de Simancas, y debajo la signatura que hemos indicado. Esta se repite en el recto de las dos primeras hojas del manuscrito y el sello en el recto de las tres.

Sorprende que en la portada del manuscrito figure como título Architetura y machinas, porque en el texto de Juan de Herrera ni una sola vez aparece el término Architetura. Además, la grafía de dicho título es distinta de la del texto de Herrera.

Estas dos observaciones nos inducen a suponer que el título fue escrito con posterioridad por otra persona ${ }^{6}$.

Probablemente quien tituló el manuscrito repasó su texto de forma somera. Por eso, luego de leer la palabra machinas repetidamente, y después de saber que estaba redactado para el monarca, con ligereza estimó que por los conocimientos de éste en architetura también trataba el texto de ella.

Estos conocimientos arquitectónicos de Felipe II ${ }^{7}$ estaban presentes en sus contemporáneos, tanto entre los arquitectos y artistas como entre los comentaristas de la época.

\footnotetext{
4 Archivo General de Simancas, Casa y Sitios Reales, Leg. 258, fol.488. Veáse el facsímil del original en Cervera Vera: El manuscrito de Juan de Herrera, 51-58.

5 Véanse los detalles de la dedicatoria en Cervera Vera: El manuscrito de Juan de Herrera, 15-16.

6 García Tapia: Ingeniería y Arquitectura, 69, erróneamente escribe el nombre de Architectura y Machinas, título que dio Juan de Herrera a un breve escrito suyo.

7 Véase "El rey y la arquitectura" en Kubler: La obra del Escorial, 37-38. Sobre sus conocimientos arquitectónicos Bury: "Las contribuciones», 334. Acerca de las numerosas fábricas que levantó en sus reinos véase Cabrera de Córdova: Filipe Segvndo, 918-922. Además Cervera Vera: El manuscrito de Juan de Herrera, 18-22.
} 
c $r$ ralos rones Lec punto a centro tradistope) quepongojed wor 4. enelpunto. 6. ae lafiguza quefesigue sinmuiau

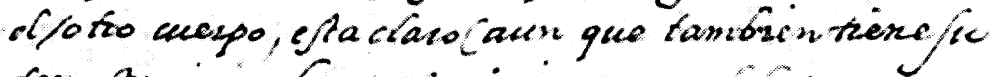
demopracion laques miminguna telar que ape

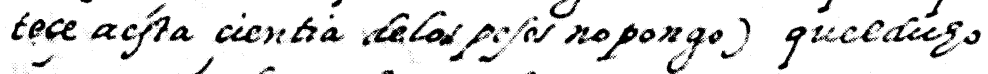
cüsepo qués el gree csta ente coxre to.c porla policion os litisi tenowapotenciá delevantar aícuezpo 6. en alto aun. quefuefe margraucquece

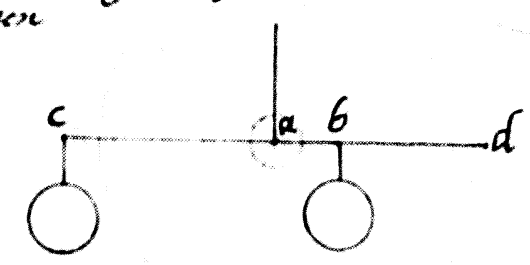

Veapues N. mat.

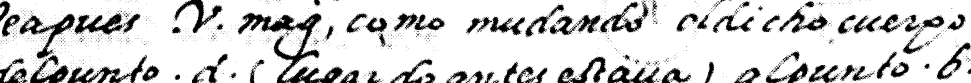
telpunto of. (Tugar do antes esiaura) a fuento.6.

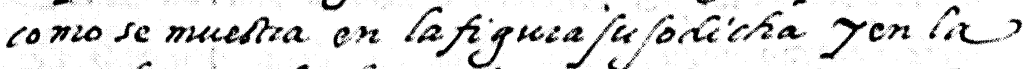
quesefigues feafor mado ina ac gapuna/ono mana nétaic mancia grec $C$ bzaco c. 6. es la a liapus má ro braco ne Zoda ia uno mana Tér cuespo.c.es alpiton orapo ton cir questa onctiab.

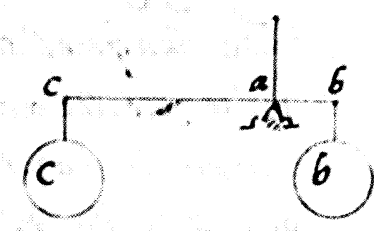
rita aljaprima yelcuenos.b. logugelevan Ea yé puento la lacapata ronde cazga la,

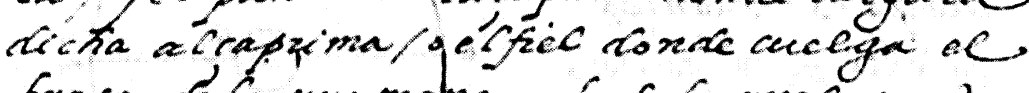
braco deía rromana ro do lo quae consta chlagiguma dichyl.

Lomefmo quefeacions A caefce crilagued,

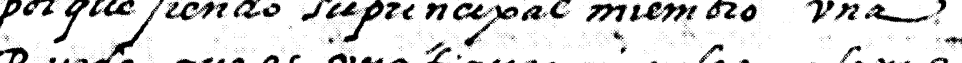
Rura que os rrafigmacircular a eme, panca ubsta queagui febec lená lata conerios.

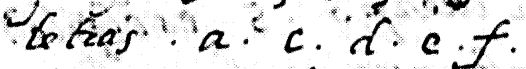
lagualefafija enviaxe Lecicita grojepa comoel

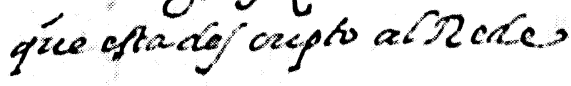

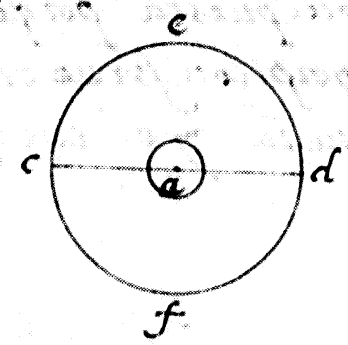

Fig. 3. Facsímil a su tamaño de la hoja 2 del manuscrito original de Herrera. 


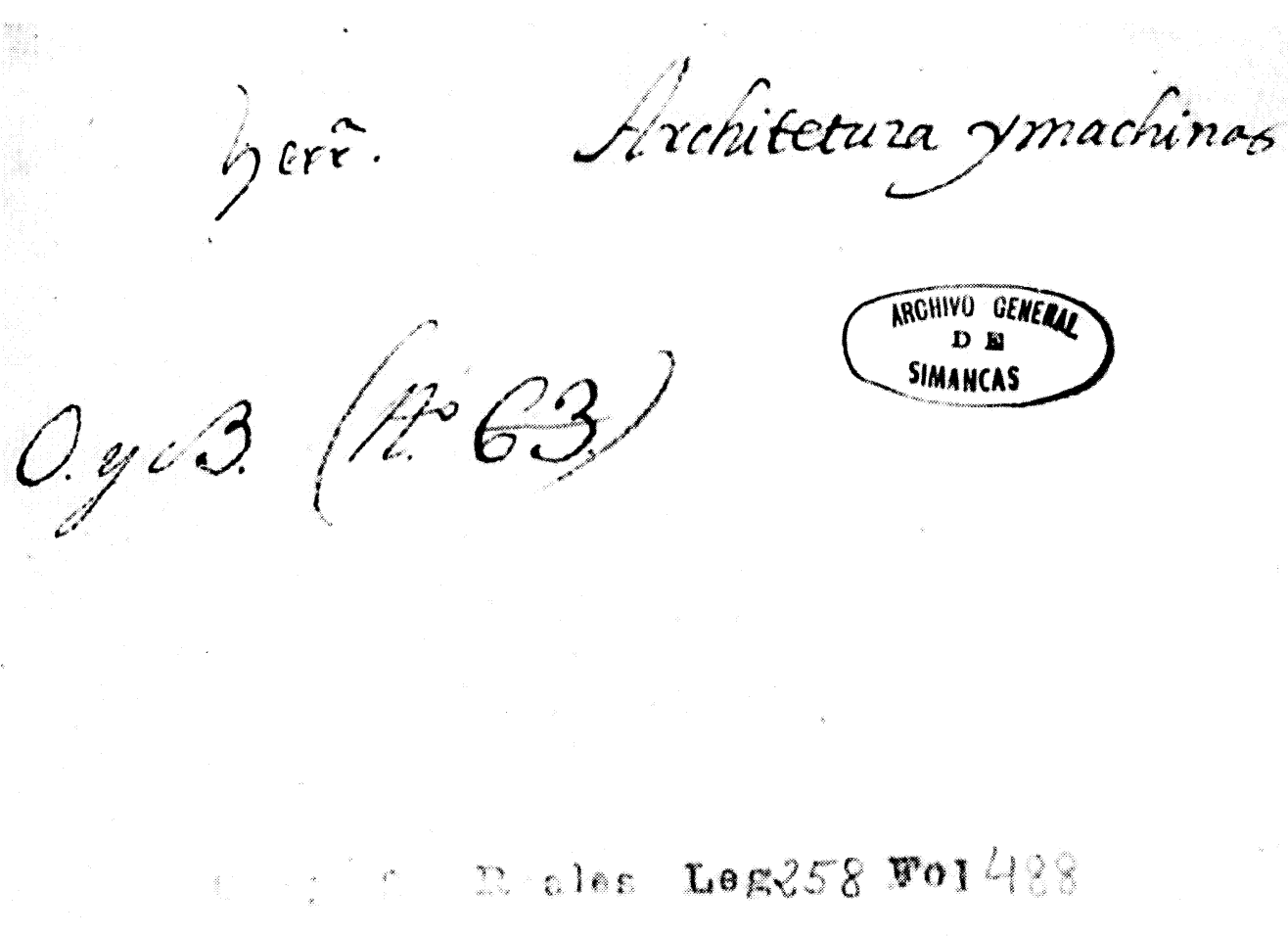

Fig. 4. Fascímil a su tamaño de la portada del manuscrito original de Herrera.

Estimamos que quien escribió Architetura, en la portada del manuscrito, estaba influido y enterado, como sus contemporáneos, del interés que prestaba Felipe II a los escritos y diseños sobre aquel arte; y por eso, sin duda, compuso el indebido título.

Por otra parte, también observamos que la caligrafía del título Architetura y machinas es distinta de la de herr. ${ }^{a}$ abreviatura indicativa de que el manuscrito estaba redactado por Juan de Herrera, y que, sin duda, fue estampada posiblemente por el Secretario real que recibió dicho texto, pues siempre llegaban al monarca los memoriales, peticiones o expedientes después de haber examinado los documentos alguno de sus Secretarios, de acuerdo con el riguroso protocolo burocrático ${ }^{8}$. Nunca los súbditos entregaban directamente a Felipe II sus documentos, porque éstos se tramitaban pausadamente, como preconizaba el método cartesiano de que los que caminan lentamente pueden llegar mucho más lejos, si van siempre por el camino recto, que los que corren, pero se apartan de él ${ }^{9}$, muy de acuerdo con la prudencia, de posible influencia germánica del monarca.

Admitiendo el año 1575 como probable fecha de la redacción del manuscrito, se puede presumir el nombre del Secretario real a quien Juan de Herrera entregó su trabajo. Fueron dos los que pudieron recibirlo: Mateo Vázquez, el Secretario favorecido por el Rey ${ }^{10}$, y Martín de Gaztelu, quien desde hacía años intervenía en los asuntos de Obras y Bosques.

8 Sobre la tarea de los secretarios véase Escudero: Los Secretarios. Como ejemplo tenemos el escrito de súplica, en el cual Juan de Herrera solicitaba alguna merced por sus trabajos extraordinarios, enviado en 1584 al Secretario real Mateo Vázquez, para que éste diera cuenta de él a Felipe II. Véase Cervera Vera: Documentos biográficos, II, 133-135, doc. 40.

9 Descartes: Discurso del método, 69.

10 Cabrera de Córdova: Filipe Segvndo, 192. 
Mateo Vázquez había sido nombrado Secretario real el 29 de marzo de 1573, sucediendo al cardenal Diego De Espinosa del que había sido Secretario y con el cual intervino en la construcción del palacio Espinosa, levantado en la villa de Martín Muñoz de las Posadas. Continuó en su oficio Vázquez hasta su fallecimiento en el año 1591. Fue amigo de Juan de Herrera y, por eso, es probable que recibiera el manuscrito en su Secretaría.

Martín de Gaztelu sucedió a Pedro de Hoyo luego de fallecer éste. Después, y a lo largo de los años, dirigió con eficacia la tramitación de los papeles relativos a las obras reales, de las que estaba encargada su Secretaría antes y con posterioridad al año 1575.

Mediante estas noticias suponemos que Juan de Herrera entregaría su manuscrito a Mateo Vázquez, tanto por su amistad con él como por la importancia que gozaba, como Secretario, ante Felipe II. Pero Mateo Vázquez, ocupado en otros menesteres de gobierno, decidió, probablemente, trasladar el manuscrito de Herrera a Martín de Gaztelu, al que consideraba como el Secretario apropiado, por dedicarse a los asuntos de Obras y Bosques y continuamente tratar del monasterio de San Lorenzo el Real.

Basamos esta suposición en el hecho, ya mencionado, de que el prior del Monasterio, fray Julián de Tricio, escribió a Martín de Gaztelu acerca de machinas y ingenios que hauia embiado Juan de Herrera, y de la respuesta favorable del monarca, como anteriormente hemos indicado.

\section{Noticias del manuscrito de Juan de Herrera}

Es probable que la primera noticia de este manuscrito se encuentre en la nota adicional de Ceán-Bermúdez a las Noticias de Llaguno, pues textualmente escribió que pudiera atribuirse a una grúa, que se presentó en aquel real sitio del Escorial para facilitar la construcción del templo y del monasterio, Juan de Herrera la describió con figuras geométricas, y la escribió de su letra, cuyo original se conserva en el real archivo de Simancas ${ }^{11}$.

En el texto de la anterior nota de Ceán-Bermúdez debió apoyarse quien escribió el manuscrito de la Colección Pedraja que comentamos. Pues resulta evidente que el título del mismo contiene las mismas palabras que escribió Ceán-Bermúdez ${ }^{12}$ y ambos no mencionan Architetura y machinas, lo cual supone que leyeron minuciosamente el texto original de Herrera.

Después, ni Menéndez Pelayo ${ }^{13}$ ni Picatoste ${ }^{14}$ mencionan el manuscrito.

En 1913 Babelon lo citó así: Aux archives de Simancas, on conserve un curieux traité de Juan de Herrera, I'architecte de I'Escorial destiné Ö expliquer au roi, par le menu, les détails de la construction, le fontionnement des appareils destinés Ö l'edification du monastäre, au montage des échafaudages ${ }^{15}$.

Ruiz de Arcaute fue quien, en 1936 y sin citar como acostumbraba su procedencia, publicó por primera vez y con figuras la transcripción del manuscrito de Simancas, aunque titulándolo Architetura y machinas, lo cual indica que no advirtió en él la inexistencia de temas arquitectónicos ${ }^{16}$.

El profesor Iñiguez Almech cuando estudió las grúas utilizadas para la construcción del monasterio de San Lorenzo el Real de El Escorial, prestó atención al manuscrito de Herrera, cuyo

\footnotetext{
11 Llaguno: Noticias, II, 125, nota 1 de Ceán-Bermúdez.

12 Véase la fig. 1 de este trabajo.

13 Menéndez Pelayo: Historia de las Ideas Estéticas, II, 372-373.

14 Picatoste: Apuntes, 153.

15 Babelon: "Gianello», 272.

16 Ruiz de Arcaute: Juan de Herrera, 36-38.
} 
texto consideró farragoso, quizá porque no comprendió sus sencillas explicaciones matemáticas. Sin embargo no mencionó el indebido título de Architetura y machinas ${ }^{17}$.

En 1917 el profesor Martín González citó el anterior trabajo de Iñiguez ${ }^{18}$. Luego, Simons y Godoy, en 1976, copiaron el texto de Ruiz de Arcaute, incluso con las figuras, titulándolo Architectura y machinas, con el error de Architectura en lugar de Architetura ${ }^{19}$.

Nosotros, en 1981, simplemente transcribimos el texto del manuscrito, con su indebido título, con objeto de dejar constancia del mismo en la biografía de Juan de Herrera ${ }^{20}$.

Posteriormente Kubler, en 1983, estima que Herrera escribió su manuscrito en 1567, fecha improbable, y elogia sus grúas y su manuscrito sin citar el falso título ${ }^{21}$.

Por último Wilkinson-Zerner cita un párrafo copiado del texto de Ruiz de Arcaute ${ }^{22}$. Y Vicente Maroto considera el manuscrito de Herrera como un Tratado teórico sobre la balanza romana o el alzaprima, y trascribe un fragmento de su primer párrafo ${ }^{23}$.

Como se puede comprobar en las anteriores referencias, sólo tres autores mencionaron como contenido del texto el de Architetura y machinas, cuyo falso título demostramos en nuestro reciente trabajo El manuscrito de Juan de Herrera indebidamente titulado "Architetura y machinas».

Con este pequeño trabajo, Archivo Español de Arte recuerda el cuatrocientos aniversario de la muerte del genial Juan de Herrera, quien entregó su alma a Dios y su cuerpo a la tierra en Madrid el 15 de enero de 1597, día de San Pablo de Tebas.

Luis Cervera Vera Real Academia de Bellas Artes de San Fernando

\section{BIBLIOGRAFÍA}

BABELON, Jean: "Gianello della Torre. Horloger de Charles-Quint et de Philippe II", en La Revue de l'Arte Ancien et Moderne, XXXIV, Paris (1913), 269-278.

Bury, J. B.: "Las contribuciones de Juan de Herrera al proyecto de El Escorial", en Goya. Revista de Arte, n. ${ }^{\circ}$ 192, Madrid (1986), 330-335.

Cabrera de Cordova, Lvis: Filipe Segvndo Rey de España, Madrid, Luis Sánchez, 1619.

Cervera Vera, Luis: Documentos biográficos de Juan de Herrera, I (1572-1581), y II (1581-1596), volúmenes I y IV de Colección de Documentos para la Historia del Arte en España, Madrid, Real Academia de Bellas Artes de San Fernando, 1981 y 1987.

-: El manuscrito de Juan de Herrera indebidamente titulado Architetura y Machinas, Valencia, Patrimonio Ediciones, S. L., 1996.

Descartes, René: Discurso del método. Traducción, estudio preliminar y notas de Risieri Frondizi, Madrid, Alianza Editorial, 1991.

Escudero, José Antonio: Los Secretarios de Estado y del Despacho (1474-1724), 2. a ed., I, II, III y IV, Madrid, Instituto de Estudios Administrativos, 1976.

GARCí TAPIA, Nicolás: Ingeniería y Arquitectura en el Renacimiento español, Valladolid, Universidad, 1990.

Iñiguez Almech, Francisco: "Los ingenios de Juan de Herrera», en El Escorial. 1563-1963. II. Arquitectura. Artes. IV Centenario de la Fundación del Monasterio de San Lorenzo el Real, Madrid, Patrimonio Nacional (1963), 181-214.

17 Iñiguez: «Los ingenios de Juan de Herrera», ed. Patrimonio, 199-202.

18 Martín González: «Noticias varias», 230.

19 Simons y Godoy: Discurso, 169-174.

20 Cervera Vera: Documentos biográficos, I, 233-235, doc. 160.

${ }_{21}$ Kubler: La obra del Escorial, 48: se le elogiaba su diligencia a la hora de proyectar máquinas o ingenios para cortas la piedra y una grúa para elevarla. El manuscrito que Herrera escribió en abril de 1567 sobre las poleas contribuyó también a asentar su reputación.

22 Wilkinson-Zerner: Juan de Herrera, 29 y 184, nota 62.

23 Vicente Maroto: "Juan de Herrera», 84. 
KubleR, George: La obra del Escorial. Versión española de Fernando Villaverde, Madrid, Alianza Editorial, 1983.

Llaguno y Amirola, Eugenio: Noticias de los arquitectos y arquitectura de España desde su restauración. Ilustradas y acrecentadas con notas, adiciones y documentos por D. Juan Agustín Cean-Bermúdez, I y II, Madrid, Imprenta Real, 1829.

Martín Gonzalez, J. J.: "Noticias varias sobre artistas de la Corte en el siglo Xvi», en Boletín del Seminario de Estudios de Arte y Arqueología, XXXV, Valladolid, Universidad (1971), 225-240.

Menéndez Pelayo, Marcelino: Historia de las Ideas Estéticas en España, II, Santander, Aldus, 1940.

Picatoste y Rodríguez, Felipe: Apuntes para una biblioteca científica española del siglo xVI, Madrid, Manuel Tello, 1891.

Ruiz de Arcaute, Agustín: Juan de Herrera. Arquitecto de Felipe II, Madrid, Espasa Calpe, 1936.

Simons, Edison y Godoy, Roberto: Discurso del Señor Juan de Herrera, aposentador Mayor de S.M., sobre la figura cúbica, Madrid, Editora Nacional, 1976.

Vicente Maroto, María Isabel: «Juan de Herrera, un hombre de ciencia», en Juan de Herrera y su influencia Actas del Simposio. Camargo, 14-17 julio 1992. Fundación Obra Pía Juan de Herrera, Universidad de Cantabria (1993), 79-89.

Wilkinson-Zerner, Catherine: Juan de Herrera. Architect to Philip II of Spain, New Haven and London, Yale University Press, 1993.

\section{NOTICIAS BIOGRÁFICAS DE UN ESCULTOR DEL SIGLO XVIII: JUAN DE LEÓN}

Al revisar la bibliografía sobre escultura del siglo xviII no es difícil encontrar citado a Juan de León cuando se mencionan algunas obras escultóricas relevantes de este siglo. Así, una y otra vez, son varios los autores que repiten su intervención en los dos importantes mausoleos reales: el de la reina M. ${ }^{a}$ Bárbara de Braganza (fig. 1 y 3) y en el de su esposo, el rey Fernando VI ${ }^{1}$, ambos en la iglesia del Monasterio de la Visitación de Madrid o Salesas Reales. Si bien no dejamos de reconocer que, por ahora, ambos sepulcros forman parte de su producción escultórica más citada y conocida no se resume únicamente en estas dos obras su labor artística. A Juan de León se le señala también como el encargado por la Junta de Fuentes del Ayuntamiento de Madrid, de labrar las esculturas que adornaban la Fuente de la Villa ${ }^{2}$ (fig. 2), obra que fue proyectada por Saqueti, hoy desaparecida, y que se adornaba con cuatro leones que arrojaban agua por la boca, sobre los que se asentaba un castillo, que sostenía como remate una figura de mujer con traje militar y un estandarte en la mano. Esta ornamentación fue hecha por el escultor en 5 meses -firmó el contrato el 17 de mayo de 1754 y se inauguró el 22 de septiembre del citado año-, haciendo manar vino por sus caños durante 15 minutos en tan señalado día.

Al proseguir revisando su labor artística volvemos a encontrarlo entre el escaso número de escultores españoles que fueron elegidos para intervenir en la decoración de la Capilla del Pala-

1 A. Ponz: Viage de España, pág. 481. Álvarez Quindos, pág. 368. E. Serrano Fatigati: Bol. Soc. Esp. Exc., t. 18, 1910, pág. 70. Polentinos, Conde de: Bol. Soc. Esp. Exc., t. 24, 1916, 281. Ceán: Dicc., t. III, pág. 10. Cruz Bahamonde: Viaje..., t. X, 540. E. Tormo: Las iglesias del antiguo Madrid, 1927. F. Portela Sandoval: La escultura en tiempos de Carlos III, Madrid, 1989. La ornamentación escultórica en la arquitectura de Sabatini en el Catálogo de la Exposición Francisco Sabatini, octubre-diciembre 1993.

2 Polentinos, Conde de: Datos históricos sobre la Casa Ayuntamiento de Madrid, Bol. Soc. Esp. Exc., t. 20, 1912, pág.

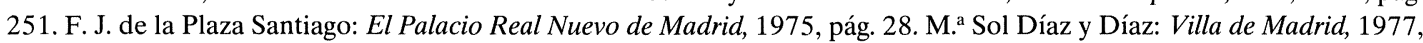
págs. $49,50$. 